\title{
Risk factors of recurrence in small-sized, node negative breast cancer in young women: a retrospective study in Chinese population
}

\author{
ZHANG XiaoSan ${ }^{1 \dagger}$, LI PengFei $^{2 \dagger}$, MA WenJie $^{1}$, DI WenYu ${ }^{3}$, ZHAO Shu ${ }^{1}$, GAO QingZu ${ }^{3}$, \\ ZHAO YuYing ${ }^{4}$, YANG MaoPeng ${ }^{1} \&$ ZHANG QingYuan ${ }^{1 *}$ \\ ${ }^{1}$ Department of Medical Oncology, the Third Affiliated Hospital of Harbin Medical University, Harbin 150081, China; \\ ${ }^{2}$ Department of Radiology, the Third Affiliated Hospital of Harbin Medical University, Harbin 150081, China; \\ ${ }^{3}$ Department of Pathology, the First Affiliated Hospital of Xin Xiang Medical College, Weihui 454100, China; \\ ${ }^{4}$ Department of Medical Oncology, the Fourth Affiliated Hospital of Harbin Medical University, Harbin 150001, China
}

Received September 17, 2012; accepted December 5, 2012; published online March 12, 2013

\begin{abstract}
We aimed to investigate risk factors of local and distant recurrence in small-sized, node negative breast cancer in women $<35$ years in a Chinese cohort. Between January 1994 and January 2007, 107 patients with pathologically confirmed small-sized $(\leqslant 1 \mathrm{~cm})$, node negative breast cancer who did not receive neoadjuvant or adjuvant chemotherapy were included. The 5-year recurrence-free survival (RFS) was estimated according to different prognostic variables. With a median time of 60 months (range, 8-60 months) follow-up, local and distant recurrence were observed in 25 cases (23.4\%). By univariate analysis, HER-2 positivity, triple negative (TN), and high Ki-67 index $(\geqslant 14 \%)$ were risk factors of a lower RFS (hazard ratio (HR) 6.680, 95\% confidence interval (CI) 2.350-18.985, $P<0.0001$ for HER-2 positive; HR 4.769, 95\%CI 1.559-14.591, $P=0.006$ for TN; HR 6.030, 95\%CI 2.659-13.674, $P<0.0001$ for high Ki-67 index). Patients with grade 3 tumors had a lower RFS (HR 2.922, 95\% CI 1.096-7.791, $P=0.032$ ) compared with those with grade 1 or grade 2 tumors. By multivariate analysis, HER-2 positivity (HR 10.204, 95\%CI 3.391-30.704, $P<0.0001$ ), TN (HR 10.521, 95\% CI 3.152-35.113, $P<0.0001$ ) and high Ki-67 index (HR 10.820, 95\% CI 4.338-27.002, $P<0.0001$ ) remained risk factors of RFS. In this cohort, HER-2 positivity, triple negative and high Ki-67 index were independent risk factors of RFS in young patients with $\mathrm{T}_{1 \mathrm{a}, \mathrm{b}} \mathrm{N}_{0}$ breast cancer. Subsequent pregnancy did not affect RFS.
\end{abstract}

breast cancer, recurrence-free survival, risk factors, young women

Citation: Zhang X S, Li P F, Ma W J, et al. Risk factors of recurrence in small-sized, node negative breast cancer in young women: a retrospective study in Chinese population. Sci China Life Sci, 2013, 56: 335-340, doi: 10.1007/s11427-013-4435-y

Breast cancer is the most common malignant tumor in women worldwide. Because of the increased use of screening mammography, the incidence of early stage breast cancer is increasing internationally [1-3]. The prognosis of small-sized $(\leqslant 1 \mathrm{~cm})$, node-negative breast cancer is generally considered to be excellent even without systemic adju-

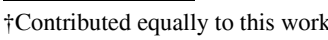

*Corresponding author (email: zhma19650210@126.com) vant therapy [4]. Nevertheless, certain subgroups of patients who are initially diagnosed with small, node-negative breast cancer have a risk of recurrence. One of the most important challenges that clinicians must face in the treatment of early breast cancer is how to distinguish those patients who will need additional systemic therapy from those patients who will not.

Some studies have identified high risk group for recur- 
rence in node-negative patients with $\leqslant 1 \mathrm{~cm}$ invasive breast cancer, and determined which patients are at the greatest risk and may benefit from adjuvant treatment. However, the results are not consistent, and few include Chinese populations.

Young women diagnosed with breast cancer have a worse disease-free survival and breast cancer specific survival than postmenopausal women. Several retrospective studies have evaluated age and prognosis; some found that young patients have a significantly higher rate of recurrence compared with their older counterpart [5,6]. Kwon et al. [5] found that age younger than 35 is an independent risk factor of recurrence, even in patients with node-negative breast cancer of $\leqslant 1 \mathrm{~cm}$. Therefore, young age of less than 35 years should be regarded as a poor prognostic factor when adjuvant therapy is considered. This is important, especially for Asian patients, because the proportion of young women in Asian countries is higher than that in Western countries $[7,8]$.

As most authors have confirmed that age younger than 35 is an independent risk factor. We narrowed our analyses to $\mathrm{T}_{1} \mathrm{~N}_{0} \mathrm{M}_{0}$ patients younger than 35 years to investigate which factors influence the outcome of this special group hoping to provide more information to guide individualized therapy in this controversial area.

Since the average age of women giving birth for the first time is increasing, many young patients diagnosed with breast cancer have not started or completed their family planning. Thus, gynecologists and oncologists are confronted more often with the question of childbearing after breast cancer. Pregnancy after a diagnosis of early stage breast cancer does not appear to increase the risk of relapse [9]. A recent meta-analysis done by Azim et al. [10] suggested that pregnancy after a diagnosis of breast cancer is associated with improved survival when compared with breast cancer patients that do not have a pregnancy after their diagnosis. To date, no study has analyzed the impact of subsequent pregnancy on recurrence-free survival (RFS) in small-sized $(\leqslant 1 \mathrm{~cm})$, node-negative breast cancer, thus we address this issue in the present article.

\section{Methods}

\subsection{Patients}

We conducted a retrospective analysis of medical records of the patients with pathologically confirmed invasive breast cancer who had received curative surgery at the Third Affiliated Hospital of Harbin Medical University, the Fourth Affiliated Hospital of Harbin Medical University, or the First Affiliated Hospital of Xinxiang Medical College from January 1994 to January 2007. Eligibility criteria included complete surgical resection, histological diagnosis of invasive ductal carcinoma of $1 \mathrm{~cm}$ or less $\left(\mathrm{T}_{1 \mathrm{a}}, \mathrm{T}_{1 \mathrm{~b}}\right)$, no lymph node metastasis $\left(\mathrm{N}_{0}\right)$, no lymphatic invasion and no venous invasion according to the 6th edition of the American Joint Committee on Cancer (AJCC) Cancer Staging Manual [11]. The number of patients with $\mathrm{T}_{1 \mathrm{mic}}$ tumors was so small that we could not include this group in our analysis. Patients with a history of previous malignancy of the breast or other sites and those who received neoadjuvant or adjuvant chemotherapy were excluded.

\subsection{Clinical and biological data acquisition}

Data on patient characteristics, type of operation and axillary lymph node examinations were collected. The type of operation was divided into mastectomy and breast conserving surgery (BCS). Pathologic information was obtained by two pathologists by reviewing pathology reports on the following variables: tumor size, histological subtype, modified Bloom-Richardson histological grade, lymphatic invasion, venous invasion and tumor border. Immunohistochemical data for standard prognostic biomarkers (ER, PR, HER-2) were also collected. For ER and PR, cases with $1 \%$ or more positive staining were considered positive. For HER-2, 3+ staining by immunohistochemistry or positivity by fluorescence in situ hybridization (FISH) was considered positive. Because most patients in our study did not have Ki-67 analysis and those with Ki-67 reports had used the old standard, we checked paraffin embedded tissue for Ki-67 and defined the high Ki-67 index using the latest standard (14\% or more positive staining as a high index) [12]. Subsequent pregnancy status of all patients was checked.

\subsection{Statistical analysis}

The median follow-up duration was 60 months (range, 8-60 months). RFS was from the date of curative surgery to the date when breast cancer recurred irrespective of locoregional or distant metastases including ipsilateral and contralateral breast recurrences or the last follow-up. RFS was estimated by the Kaplan-Meier method. The log-rank test was used for a comparison of the survival curves between subgroups according to prognostic factors. Cox proportional hazards model was used to determine association of breast cancer subtypes with other covariates. $P$-value $<0.05$ was considered significant.

All statistical analyses were performed using SPSS for Windows software, version 19.0 (SPSS Inc, Chicago, IL).

\section{Results}

We identified 159 patients with a primary tumor size of $1 \mathrm{~cm}$ or less and age $<35$ years. After excluding 47 patients who received neoadjuvant or adjuvant chemotherapy, 3 patients lost to follow-up and 2 patients who died from other reasons (not related to tumor), 107 patients were included in the analysis. 
Patient characteristics are shown in Table 1. The median age at diagnosis of this cohort was 32 years (range, 19-34 years), of which $27(25.2 \%)$ were younger than 30 years and $80(74.8 \%)$ were in the $\geqslant 30$ and $<35$ years group. Of the 107 patients, 34, 45 and 28 had grade 1, 2 and 3 tumors, respectively. As for cancer type, $58(54.2 \%)$ patients had hormone receptor (HR) positive tumors (ER+ and/or $\mathrm{PR}+$ / HER-2+,-), 26 (24.3\%) had HER-2 positive tumors (HER-2+/ER-/PR-), and $23(21.5 \%)$ had triple negative breast cancer (TNBC) (ER-/PR-/HER-2-). As for the tumor stage, $50(46.7 \%)$ and $57(53.3 \%)$ had $\mathrm{T}_{1 \mathrm{a}}$ and $\mathrm{T}_{1 \mathrm{~b}}$ tumors, respectively. Regarding treatment, 95 (88.8\%) patients underwent mastectomy and $12(11.2 \%)$ patients received BCS+radiotherapy. There was no difference in surgery choice between $\mathrm{T}_{1 \mathrm{a}}$ and $\mathrm{T}_{1 \mathrm{~b}}$ groups. In Ki-67 analysis, $30(28 \%)$ patients had a high Ki-67 index while $77(72 \%)$ patients had a low Ki-67 index. Almost all patients (86.2\%) with HR positive tumors received adjuvant hormonal therapy. Subsequent pregnancy was found in 13 (12.1\%) patients. Table 2 shows the 5 year RFS by different characteristics and HR value of different risk factors according to univariate analysis.

By univariate analysis, HER-2 positivity, TN and high Ki-67 index $(\geqslant 14 \%)$ were risk factors of a lower RFS (HR 6.680, 95\%CI 2.350-18.985, $P<0.0001$ for HER-2 positive; HR 4.769, 95\%CI 1.559-14.591, $P=0.006$ for TN; HR $6.030,95 \%$ CI $2.659-13.674, P<0.0001$ for high Ki-67 index). Patients with grade 3 tumors had a lower RFS (HR 2.922, 95\%CI 1.096-7.791, $P=0.032$ ) compared with those with grade 1 or grade 2 tumors. Age $(<30$ years versus $\geqslant 30$ years), tumor size $\left(\mathrm{T}_{1 \mathrm{a}}\right.$ versus $\left.\mathrm{T}_{1 \mathrm{~b}}\right)$, treatment mode and subsequent pregnancy did not affect RFS in this cohort. Differences in RFS by different pathological subtype, Ki-67 index and grade groups are shown in Figures 1, 2, and 3, respectively.

By multivariate analysis, HER-2 positive (HR 10.204, 95\%CI 3.391-30.704, $P<0.0001$ ), TN (HR 10.521, 95\%CI $3.152-35.113, P<0.0001)$ and high Ki-67 index (HR 10.820, 95\%CI 4.338-27.002, $P<0.0001)$ remained risk factors of RFS. Grade 3 did not reach statistical significance (HR $2.129,95 \%$ CI $0.776-5.837, P=0.142)$. Results of multivariate analysis are shown in Table 3.

Table 1 Distribution of 107 breast cancer cases according to selected individual characteristics

\begin{tabular}{|c|c|c|}
\hline Characteristics & $n$ & $\%$ \\
\hline \multicolumn{3}{|l|}{ Age at diagnosis } \\
\hline$<30$ & 27 & 25.2 \\
\hline$\geqslant 30$ and $<35$ & 80 & 74.8 \\
\hline \multicolumn{3}{|l|}{$\mathrm{T}$} \\
\hline T1a & 50 & 46.7 \\
\hline $\mathrm{T} 1 \mathrm{~b}$ & 57 & 53.3 \\
\hline \multicolumn{3}{|l|}{ Grade } \\
\hline 1 & 34 & 31.8 \\
\hline 2 & 45 & 42.1 \\
\hline 3 & 28 & 26.1 \\
\hline \multicolumn{3}{|l|}{ Breast cancer subtypes $^{\text {a) }}$} \\
\hline $\mathrm{HR}+(\mathrm{ER}+$ and/or PR+/HER-2+,- $)$ & 58 & 54.2 \\
\hline HER-2+ (HER-2+/ER-/PR-) & 26 & 24.3 \\
\hline TNBC (ER-/PR-/HER-2-) & 23 & 21.5 \\
\hline \multicolumn{3}{|l|}{ Surgery } \\
\hline Mastectomy & 95 & 88.8 \\
\hline $\mathrm{BCS}+\mathrm{RT}^{\mathrm{b})}$ & 12 & 11.2 \\
\hline \multicolumn{3}{|l|}{ Adjuvant hormonal therapy (HT) } \\
\hline None & 8 & 13.8 \\
\hline HT & 50 & 86.2 \\
\hline \multicolumn{3}{|l|}{ Ki-67 } \\
\hline$<14 \%$ & 77 & 72.0 \\
\hline$\geqslant 14 \%$ & 30 & 28.0 \\
\hline \multicolumn{3}{|l|}{ Subsequent pregnancy } \\
\hline No & 94 & 87.9 \\
\hline Yes & 13 & 12.1 \\
\hline
\end{tabular}

a) ER, estrogen receptor; PR, progesterone receptor; HER-2+, human epidermal growth factor receptor 2; TNBC, triple negative breast cancer; b) $\mathrm{BCS}+\mathrm{RT}$, breast conserving surgery+radiotherapy. 
Table 2 Five years recurrence-free survival by different characteristics and HR value of different risk factors in univariate analysis ${ }^{\text {a) }}$

\begin{tabular}{|c|c|c|c|c|}
\hline Characteristics & Patients at risk $(n)$ & Recurrence $(n)$ & $P$ & HR $(95 \% \mathrm{CI})$ \\
\hline \multicolumn{5}{|l|}{ Age at diagnosis } \\
\hline$<30$ & 27 & 8 & & \\
\hline$\geqslant 30$ and $<35$ & 80 & 17 & 0.398 & $0.696(0.300-1.613)$ \\
\hline \multicolumn{5}{|l|}{$\mathrm{T}$} \\
\hline T1a & 50 & 11 & & \\
\hline $\mathrm{T} 1 \mathrm{~b}$ & 57 & 14 & 0.674 & $1.185(0.538-2.610)$ \\
\hline 1 & 34 & 6 & & \\
\hline 2 & 45 & 7 & 0.763 & $0.845(0.284-2.516)$ \\
\hline 3 & 28 & 12 & 0.032 & $2.922(1.096-7.791)$ \\
\hline \multicolumn{5}{|l|}{ Breast cancer subtypes } \\
\hline $\mathrm{HR}+(\mathrm{ER}+$ and/or PR+/HER-2+,- $)$ & 58 & 5 & & \\
\hline HER-2+ (HER-2+/ER-/PR-) & 26 & 12 & $<0.0001$ & $6.680(2.350-18.985)$ \\
\hline \multicolumn{5}{|l|}{ Surgery } \\
\hline Mastectomy & 95 & 20 & & \\
\hline $\mathrm{BCS}+\mathrm{RT}$ & 12 & 5 & 0.192 & $1.922(0.721-5.123)$ \\
\hline \multicolumn{5}{|l|}{ Adjuvant hormonal therapy (HT) } \\
\hline None & 8 & 1 & & \\
\hline HT & 50 & 4 & 0.677 & $0.627(0.070-5.616)$ \\
\hline \multicolumn{5}{|l|}{$\mathrm{Ki}-67$} \\
\hline$<14 \%$ & 77 & 9 & & \\
\hline$\geqslant 14 \%$ & 30 & 16 & $<0.0001$ & $6.030(2.659-13.674)$ \\
\hline \multicolumn{5}{|l|}{ Subsequent pregnancy } \\
\hline No & 94 & 21 & & \\
\hline Yes & 13 & 4 & 0.464 & $1.492(0.512-4.347)$ \\
\hline
\end{tabular}

a) Abbreviations as in Table 1 .

Table 3 Multivariate analysis results

\begin{tabular}{ccc}
\hline Variables & HR $^{\text {a) }}\left(95 \%\right.$ CI $\left.^{\text {b) }}\right)$ & \\
\hline HER-2 positive & $10.204(3.391-30.704)$ & $<0.0001$ \\
Triple negative & $10.521(3.152-35.113)$ & $<0.0001$ \\
High Ki-67 $(\geqslant 14 \%)$ & $10.820(4.338-27.002)$ & $<0.0001$ \\
Grade 3 & $2.129(0.776-5.837)$ & 0.142 \\
\hline
\end{tabular}

a) HR, hazard ratio; b) CI, confidence interval.

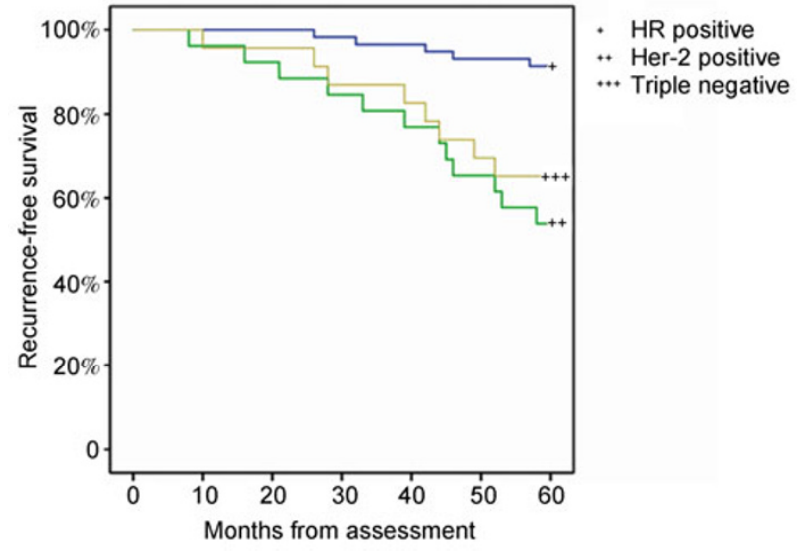

Figure 1 Five years recurrence-free survival by different breast cancer subtype.

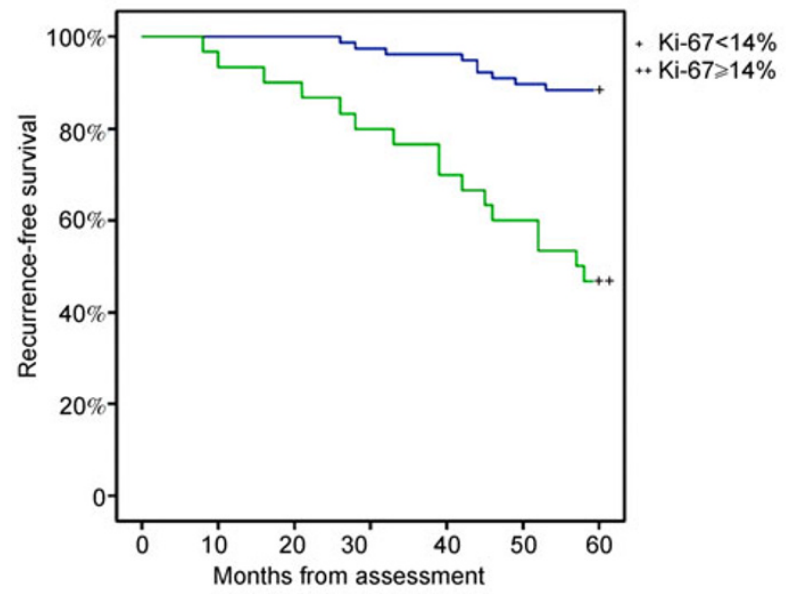

Figure 2 Five years recurrence-free survival by different Ki-67 index. 


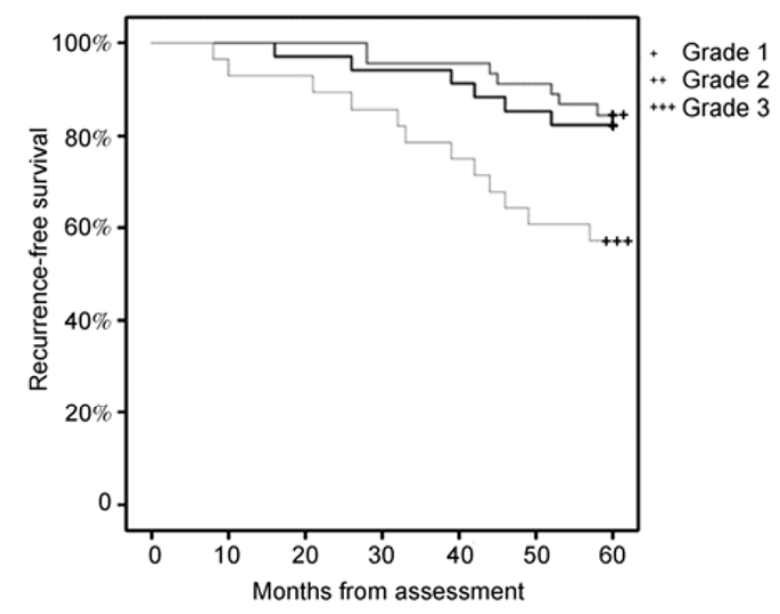

Figure 3 Five years recurrence-free survival by different histologic grade.

\section{Discussion}

In the present study, we found that there was no difference in RFS between mastectomy and BCS+radiotherapy groups. This is consistent with a retrospective study of Moroccan young women by Abahssain et al. [13]. Although the NCCN guidelines refer to age $<35$ years as a relative contraindication for BCS, BCS+radiotherapy seems to be a reasonable local treatment choice for early breast cancer in patients $<35$ years. The reason for this is probably because our research subjects were patients with small tumors and small-sized $(\leqslant 1 \mathrm{~cm})$, node-negative breast cancer. This is a special group which has been reported to have an excellent prognosis [4]. Prospective studies are needed to evaluate the role of BCS in patients $<35$ years.

According to the 2010 National Comprehensive Cancer Network Guidelines, small tumors (those less than $0.5 \mathrm{~cm}$ in greatest diameter $\left(\mathrm{T}_{1 \mathrm{a}}\right)$ that do not involve the lymph nodes are so favorable that adjuvant systemic therapy is of minimal benefit and is not recommended. These guidelines also state that patients with invasive breast cancer $0.6-1 \mathrm{~cm}$ in diameter and no lymph node involvement $\left(\mathrm{T}_{1 \mathrm{~b}}\right)$ may be divided into patients with a low risk of recurrence and those with unfavorable prognostic features (intramammary angiolymphatic invasion, high nuclear grade, high histological grades, HER-2 positive status, HR negative status) warrant consideration for adjuvant chemotherapy [14].

Studies have shown that certain biologic subtypes, including HER-2 positive and TNBC tumors, have an increased risk of relapse, despite their small size, even less than $0.5 \mathrm{~cm}$ in greatest diameter $\left(\mathrm{T}_{1 \mathrm{a}}\right)$ [15-19]. The present study showed that HER-2 positivity and TN status were correlated with worse RFS. There were no RFS differences between the $T_{1 a}$ and $T_{1 b}$ groups. Patients who have HER-2 positive or triple negative early breast cancer may need adjuvant chemotherapy, even with tumors of less than $0.5 \mathrm{~cm}$ in size $\left(\mathrm{T}_{1 \mathrm{a}}\right)$. We also identified that grade was an independent risk factor in the univariate analysis, but it did not reach statistical significance in the multivariate analysis. This is consistent with results of Kwon et al. [5] and Albert et al. [17]. Some authors reported that a high grade of tumor is associated with a high relapse or mortality rate [20-22]. The influence of tumor grade on outcome needs further investigation.

The results on $\mathrm{Ki}-67$ as a risk factor of local recurrence show discrepancy. Kwon et al. [5] pointed out that Ki-67 did not affect RFS, while Colleoni et al., in a study of 425 patients, found that in both univariate and multivariate analyses the most relevant prognostic factor of local and distant relapse for this low-risk population was Ki-67 labeling [23]. The reason for this discrepancy is possibly that they used a different standard for the high Ki-67 index (Kwon et al. used $\geqslant 10 \%$ while Colleoni et al. used $\geqslant 20 \%$ of the cells as the high ki-67 index). To our knowledge, we are the first to investigate $\mathrm{Ki}-67$ as a risk factor of RFS in this special group using the latest standard $(\geqslant 14 \%$ of the cells as high Ki-67 index). We found that Ki-67 was also an independent risk factor of RFS. For patients with a high Ki-67 index and even for HR positive patients, chemotherapy should be considered.

In the present study, we found no difference in RFS in the pregnancy and non-pregnancy groups. Young patients who have not started or completed their family planning may have a strong desire to have a child, especially for those with early stage breast cancer. To our knowledge, we are the first to investigate the impact of subsequent pregnancy on RFS in small-sized $(\leqslant 1 \mathrm{~cm})$, node-negative breast cancer and our result may help to give such patients confidence to become a mother after the diagnosis of breast cancer.

The role of trastuzumab (Herceptin) in early stage Her-2 positive breast cancer in young women is of great importance. Herceptin is usually used together with chemotherapy. Because we chose patients who did not receive chemotherapy for the current study, we can not address this issue.

In our cohort, most HR positive patients received adjuvant hormonal therapy which is the routine treatment for such patients. Adjuvant hormonal therapy was analyzed as an independent risk factor in the univariate analysis; however, it did not seem to influence RFS. The reason for this may be the relatively small size of this subgroup. Also, our follow-up time was only 60 months and the recurrence rate in the early stage HR positive group is very low even without adjuvant hormonal therapy. To investigate this question, we need to expand the sample size and prolong the followup time.

Several limitations of this study should be considered when interpreting the results. First, this is a retrospective study which lends inherent bias. Second, we used the path- 
ological data of different institutions in the analysis and this may lead to bias because of differing quality-control procedures in different hospitals even though we investigated all of the pathological results by two pathologists.

\section{Conclusion}

In practice, many early stage breast cancer patients receive chemotherapy simply because they are younger than 35 years. However, some patients with a good prognosis may not need chemotherapy. In the present study, we identified that HER-2 positivity, TNBC and high Ki-67 were independent risk factors for RFS in young small-sized $(\leqslant 1 \mathrm{~cm})$, node-negative breast cancer patients. Patients with HER-2 positivity, TNBC and high Ki-67 tumors should be considered to receive chemotherapy. BCS+radiotherapy may be a reasonable local treatment choice for early breast cancer patients even for those aged $<35$ years, although this needs further confirmation. There is no difference in RFS between the very young $(<30$ years) and the young $(\geqslant 30$ and $<35$ years). $T_{1 a}$ and $T_{1 b}$ have no difference in RFS, so we need not treat them differently. Subsequent pregnancy and adjuvant hormonal therapy may not affect RFS in young early stage breast cancer patients although confirmation will be necessary by a large sample study.

1 Otto S J, Fracheboud J, Looman C W, et al. Initiation of population-based mammography screening in Dutch municipalities and effect on breast-cancer mortality: a systematic review. Lancet, 2003, 361: 1411-1417

2 Berry D A, Cronin K A, Plevritis S K, et al. Effect of screening and adjuvant therapy on mortality from breast cancer. N Engl J Med, 2005, 353: 1784-1792

3 Duijm L E, Groenewoud J H, Roumen R M, et al. A decade of breast cancer screening in The Netherlands: trends in the preoperative diagnosis of breast cancer. Breast Cancer Res Treat, 2007, 106: 113-119

4 Joensuu H, Pylkkanen L, Toikkanen S. Late mortality from $\mathrm{pT}_{1} \mathrm{~N}_{0} \mathrm{M}_{0}$ breast carcinoma. Cancer, 1999, 85: 2183-2189

5 Kwon J H, Kim Y J, Lee K W, et al. Triple negativity and young age as prognostic factors in lymph node-negative invasive ductal carcinoma of $1 \mathrm{~cm}$ or less. BMC Cancer, 2010, 10: 557

6 Theriault R L, Litton J K, Mittendorf E A, et al. Age and survival estimates in patients who have node-negative $\mathrm{T}_{\text {lab }}$ breast cancer by breast cancer Subtype. Clin Breast Cancer, 2011, 11: 5, 325-331
7 Ahn S H. Clinical characteristics of breast cancer patients in Korea in 2000. Arch Surg, 2004, 139: 27-30

8 Ahn S H, Son B H, Kim S W, et al. Poor outcome of hormone- receptor positive breast cancer at very young age is due to tamoxifen resistance: nationwide survival data in Korea- a report from the Korean Breast Cancer Society. J Clin Oncol, 2007, 25: 2360-2368

9 Rippy E E, Karat I F, Kissin M W. Pregnancy after breast cancer: the importance of active counselling and planning. Breast, 2009, 18: $345-350$

10 Azim H A Jr, Santoro L, Pavlidis N, et al. Safety of pregnancy following breast cancer diagnosis: a meta-analysis of 14 studies. Eur J Cancer, 2011, 47: 74-83

11 Cancer AJCo AJCC Cancer Staging Manual. New York: Springer, 2002. 6

12 Voduc K D, Cheang M C, Tyldesley S, et al. Breast cancer subtypes and the risk of local and regional relapse. J Clin Oncol, 2010, 28: 1684-1691

13 Abahssain H, Lalya I, El M'Rabet F Z, et al. Breast cancer in Moroccan young women: a retrospective study. BMC Res Notes, 2010, 3: 286

14 Chen A M, Meric-Bernstam F, Hunt K K, et al. Breast conservation after neoadjuvant chemotherapy. Cancer, 2005, 103: 689-695

15 Gonzalez-Angulo A M, Litton J K, Broglio K R, et al. High risk of recurrence for patients with breast cancer who have human epidermal growth factor receptor 2-positive, node-negative tumors $1 \mathrm{~cm}$ or smaller. J Clin Oncol, 2009, 27: 5700-5706

16 Amar S, McCullough A E, Tan W, et al. Prognosis and outcome of small $(<=1 \mathrm{~cm})$, node-negative breast cancer on the basis of hormonal and HER-2 status. Oncologist, 2010, 15: 1043-1049

17 Albert J M, Gonzalez-Angulo A M, Guray M, et al. Estrogen/progesterone receptor negativity and HER-2 positivity predict locoregional recurrence in patients with $\mathrm{T}_{1 \mathrm{a}, \mathrm{b}} \mathrm{N}_{0}$ breast cancer. Int $\mathrm{J}$ Radiat Oncol Biol Phys, 2010, 77: 1296-1302

18 Park Y H, Kim S T, Cho E Y, et al. A risk stratification by hormonal receptors $(\mathrm{ER}, \mathrm{PgR})$ and HER-2 status in small $(<$ or $=1 \mathrm{~cm})$ invasive breast cancer: who might be possible candidates for adjuvant treatment? Breast Cancer Res Treat, 2010, 119: 653-661

19 Joensuu $\mathrm{H}$, Isola $\mathrm{J}$, Lundin $\mathrm{M}$, et al. Amplification of erbB2 and erbB2 expression are superior to estrogen receptor status as risk factors for distant recurrence in $\mathrm{pT}_{1} \mathrm{~N}_{0} \mathrm{M}_{0}$ breast cancer: a nationwide population-based study. Clin Cancer Res, 2003, 9: 923-930

20 Hanrahan E O, Gonzalez-Angulo A M, Giordano S H, et al. Overall survival and cause-specific mortality of patients with stage $\mathrm{T}_{1 \mathrm{a}, \mathrm{b}} \mathrm{N}_{0} \mathrm{M}_{0}$ breast carcinoma. J Clin Oncol, 2007, 25: 4952-4960

21 Chia S K, Speers C H, Bryce C J, et al. Ten-year outcomes in a population-based cohort of node-negative, lymphatic, and vascular invasion-negative early breast cancers without adjuvant systemic therapies. J Clin Oncol, 2004, 22: 1630-1637

22 Soerjomataram I, Louwman M W, Ribot J G, et al. An overview of prognostic factors for long-term survivors of breast cancer. Breast Cancer Res Treat, 2008, 107: 309-330

23 Colleoni M, Rotmensz N, Peruzzotti G, et al. Minimal and small size invasive breast cancer with no axillary lymph node involvement: the need for tailored adjuvant therapies. Ann Oncol, 2004, 15: 16331639

Open Access This article is distributed under the terms of the Creative Commons Attribution License which permits any use, distribution, and reproduction in any medium, provided the original author(s) and source are credited. 